\title{
Racial Variation in the Cost-Effectiveness of Chemotherapy for Prostate Cancer
}

\author{
By Michael Grabner, PhD, Eberechukwu Onukwugha, PhD, Rahul Jain, PhD, and C. Daniel Mullins, PhD
}

University of Maryland School of Pharmacy, Baltimore, MD; and University of Georgia College of Pharmacy, Athens, GA

\begin{abstract}
Purpose: Heterogeneity of treatment effects and expenditures impacts the cost-effectiveness of health interventions. This study investigates the variation in costs, effects, and incremental costeffectiveness ratios (ICERs) associated with chemotherapy in elderly patients with metastatic (M1) prostate cancer (PC) across race/ethnicity subgroups (non-Hispanic whites, non-Hispanic blacks, and others).
\end{abstract}

Study Design: Retrospective observational analysis.

Methods: We examined patients age 66 years or older, identified by using the linked Surveillance, Epidemiology, and End Results-Medicare data set, who were diagnosed with M1 PC between 2000 and 2005. Cost data on the basis of Medicare reimbursements were available for 36 months after diagnosis. Mean costs and effects (life-years gained [LYG]) were adjusted for censoring. The baseline scenario examined PC-specific medical costs at 24 months and required survival of at least three months. Sensitivity analysis considered sampling uncertainty,

\section{Introduction}

Accounting for heterogeneity of treatment effects is a cornerstone of evidence-based medicine. ${ }^{1}$ As a result, clinical trials increasingly aim to test for effect differences across population subgroups. ${ }^{2}$ In addition to heterogeneity of treatment effects, economic evaluations of health interventions also need to consider variation in health care expenditures across subgroups. ${ }^{3}$ Variation in treatment effects, costs, or both will lead to variation in incremental cost-effectiveness ratios (ICERs); a given intervention may therefore be cost-effective for some subgroups but not for others. ${ }^{4}$ This suggests that subgroup analysis is particularly informative for conducting cost-effectiveness studies for payers with varying proportions of enrollee subgroups. Insurance companies increasingly request information reflective of their member populations to be able to design better coverage and reimbursement schemes.

In the prostate cancer (PC) setting, the existence of racial disparities in access to health care and in treatment outcomes constitutes another rationale for the conduct of subgroup analysis. PC mortality rates are more than twice as high in black men compared with white men in the United States. ${ }^{5}$ This difference may result from racial disparities in health services use; compared with whites, blacks are more likely to be diagnosed with metastatic disease ${ }^{6}$ but less likely to undergo PC screening $^{7}$ and to receive aggressive treatment rather than watchful waiting. ${ }^{8}$ Differences in tumor biology, health knowledge, and relationships with care providers may also play a role. ${ }^{9,10}$ selection into treatment, and adjustments to initial model assumptions.

Results: We identified 3,888 patients with M1 PC, of whom $24 \%$ ( $n=930$ ) received chemotherapy (primarily docetaxel and mitoxantrone). Twenty percent of observations were censored. The full sample ICER was \$99,146 per LYG (95\% CI, \$75,042 to $\$ 130,195)$. Estimates for whites (ICER, \$107,095; 95\% Cl, $\$ 78,391$ to $\$ 148,272$ ), blacks (ICER, $\$ 59,887 ; 95 \% \mathrm{Cl}$, $\$ 22,860$ to $\$ 121,509$ ), and others (ICER, $\$ 123,909 ; 95 \% \mathrm{Cl}, \$ 37,782$ to $\$ 366,376$ ) suggest considerable variation in the likelihood of chemotherapy being cost-effective. Results were similar in sensitivity analysis.

Conclusion: Chemotherapy use in elderly patients with $\mathrm{M} 1$ PC is associated with an ICER of $\$ 99,146$ per LYG. Subgroup analysis revealed heterogeneity in point estimates and considerable statistical uncertainty. To generate a reliable evidence base, efforts to increase the representation of minorities in health care data sets need to continue.

These motivations for subgroup analysis are important to consider when estimating real-world cost-effectiveness of chemotherapy in patients with advanced-stage PC, a setting in which variation in ICERs across subgroups has not been previously explored. Chemotherapy in patients with advanced-stage PC was restricted to palliative care for most of the previous decade. ${ }^{11}$ In 2004, docetaxel-based chemotherapy showed a survival benefit compared with mitoxantrone-based chemotherapy and was approved by the Food and Drug Administration for the treatment of metastatic hormone-refractory PC. ${ }^{12}$ Several trials examining a variety of new chemotherapy agents for second-line therapy are currently under way. ${ }^{13,14}$ These developments suggest that chemotherapy will play an increasingly larger role in the management of patients with advanced-stage PC. At the same time, chemotherapy costs have been steadily increasing; the median price of cancer drugs rose from $\$ 1,553$ in 1995 to 1999 to $\$ 7,112$ in 2005 through 2009 (in 2007 dollars). ${ }^{15}$ Optimal allocation of health care resources warrants an investigation into the cost-effectiveness of these treatments, given their moderate survival benefits and substantial costs.

The objective of this study is to explore variation in the cost-effectiveness of chemotherapy for advanced-stage PC across demographic groups defined by race/ethnicity. We compare patients who received any type of chemotherapy with those who did not receive chemotherapy and present results for subgroups as well as for the full sample. We also adjust our cost estimates for the presence of censoring and conduct various types of sensitivity analyses. 


\section{Methods}

\section{Data Source and Study Population}

This retrospective observational study used patient-level data from the linked Surveillance, Epidemiology and End Results (SEER) -Medicare claims database. We examined patients diagnosed with incident PC between 2000 and 2005 with claims available from 1999 until the end of the study period (December 2007). Enrollment was limited to those patients who received androgen deprivation therapy at any time during observation and who had been diagnosed with metastatic (M1) disease as defined using American Joint Committee on Cancer-TNM staging criteria. ${ }^{16}$ Exclusion criteria are listed in the Appendix.

Patients were censored if they enrolled in a health maintenance organization or lost Part A and/or B enrollment at any time after diagnosis. This study was approved by the University of Maryland Baltimore institutional review board (HP00042760).

\section{Estimation of Costs and Effects}

The study takes a payer perspective. Costs were derived as Medicare payments to providers (reimbursements in \$US), which generally are considered a good proxy for true economic costs. ${ }^{17}$ Prescription drug payment information from Medicare Part D is not yet available in SEER-Medicare, but Part B covers most types of chemotherapy and related treatments. ${ }^{17}$ Monthly costs were available for a maximum of 36 months after diagnosis and summed up over time, until death or censoring occurred, to calculate final costs per patient. The baseline analysis considered all medical care costs related to a PC diagnosis, given that these costs are most likely to differ across PC patients who received or who did not receive chemotherapy.

As a result of skewed cost data, we used a nonparametric bootstrap with 1,000 repetitions to test for differences across groups. ${ }^{18,19}$ We also addressed the fact that cost observations are right-censored because of patients dropping out of the sample for reasons other than death-for instance, by enrolling in a health maintenance organization. We adjusted for censoring by using an inverse probability weighting technique proposed by Bang and Tsiatis, ${ }^{20,21}$ which uses only completely observed patients but, unlike simpler alternative approaches, corrects for the disproportionate influence of patients with shorter survival times. The method accomplishes this adjustment by scaling down the cost contribution of patients who are less likely to be censored and scaling up the cost contribution of patients who are more likely to be censored.

Effectiveness was measured using mean survival improvement in days from diagnosis, estimated as the area under the Kaplan-Meier survival curve during the relevant time horizon. Chemotherapy use was defined as the presence of a claim for any chemotherapy agent based on the Healthcare Common Procedures Coding System (covering hospital inpatient, outpatient, home health care, and hospice claims) and the $\mathrm{Na}$ tional Drug Code number (covering durable medical equipment providers).
ICERs were calculated as the difference in mean costs across treatment (any chemotherapy) and comparison (no chemotherapy) groups divided by the difference in mean effectiveness (life-years gained [LYG]). CIs for the ICER were derived using a percentile-based nonparametric bootstrap with 1,000 repetitions. ${ }^{22}$

\section{Primary Statistical Analysis}

The baseline scenario used a time horizon of 24 months from diagnosis for the calculation of costs and effects and limited the study population to those patients who survived at least three months after diagnosis. No discounting or inflation-adjustment was implemented. All calculations were performed for the full sample as well as for subgroups defined by race/ethnicity (nonHispanic whites, non-Hispanic blacks, others). Statistical analysis was performed using SAS version 9.2 (SAS Institute, Cary, North Carolina). Estimates were considered statistically significant if the associated $P$ value was less than .05 .

\section{Sensitivity Analysis}

Sensitivity analysis explored the effects of changes on the estimated ICER ( $v$ baseline): all medical care costs ( $v$ PC-specific medical care costs), a 36-month time horizon from diagnosis ( $v$ a 24-month horizon), and no survival requirement ( $v$ a requirement of at least three months of survival). Sampling uncertainty was evaluated graphically using an ICER scatter plot and a cost-effectiveness acceptability curve (CEAC) on the basis of 1,000 repetitions that were generated by using the bootstrap technique. A CEAC plots the probability of the treatment being cost-effective for a continuum of willingness-to-pay (WTP) thresholds. ${ }^{23}$

We also calculated ICERs in propensity score (PS) - matched samples, stratified by subgroups, to account for selection into treatment on the basis of observable patient characteristics. ${ }^{24}$ Patients were matched based on their propensity to receive chemotherapy

\section{Take-Away Points}

Chemotherapy in patients with metastatic prostate cancer may be cost-effective for some racial subgroups but not for others. Considerable uncertainty presents challenges for managed-care decision makers.

- Point estimates of incremental cost-effectiveness ratios range from $\$ 60,000$ per life-year gained (LYG) for blacks to $\$ 107,000$ per LYG for whites and $\$ 124,000$ per LYG for others (Asians and Hispanics). Estimates for minorities are associated with substantial statistical uncertainty.

- Depending on an insurance company's enrollee population characteristics, this uncertainty may lead to different coverage and reimbursement decisions.

- Efforts to increase the representation of minorities in health care data sets need to continue. 


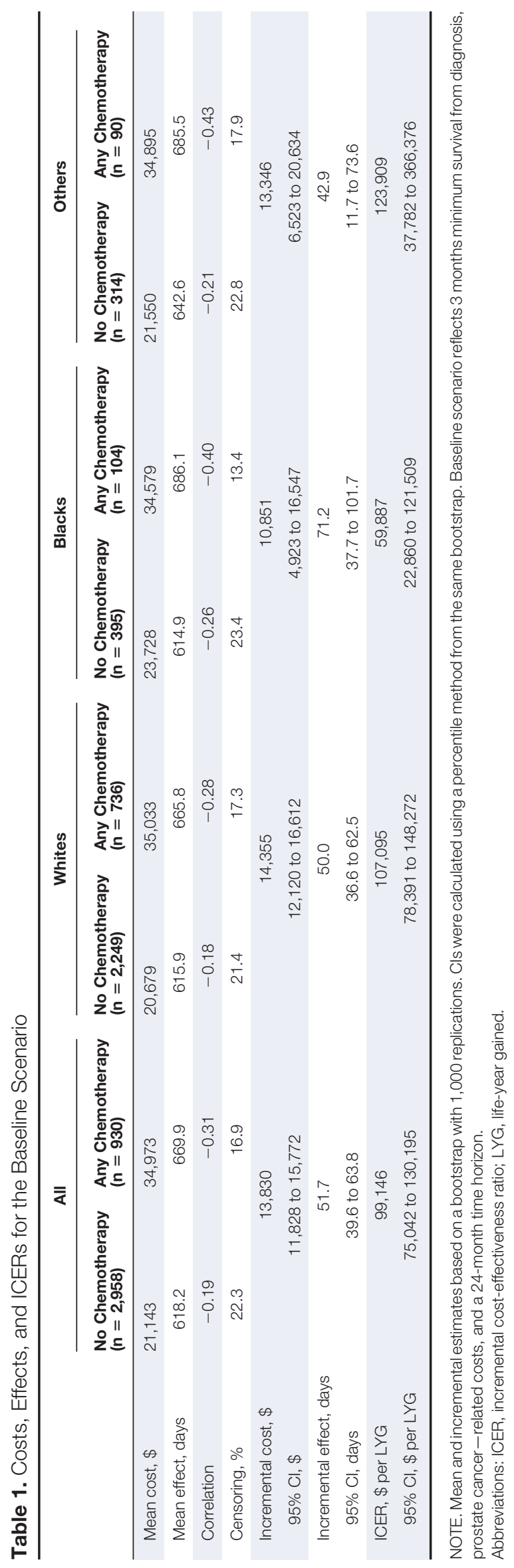


by using a greedy matching technique for each subgroup. ${ }^{25} \mathrm{~A}$ list of variables for the PS model is available in the Appendix.

\section{Results}

\section{Descriptive Results}

We identified 3,888 patients with M1 PC who were on androgen deprivation therapy during the sample period and who survived three months or more after diagnosis. The race distribution of non-Hispanic whites, non-Hispanic blacks, and others was $77 \%, 13 \%, 10 \%$, respectively. Average age was 78 years (standard deviation, 7 years), and median follow-up was 26 months (average, 31 months). Twenty-four percent of patients $(n=930)$ received chemotherapy at any time during follow-up. Docetaxel-based regimens accounted for $72 \%$ of chemotherapy claims $(62 \%$ in $2000 ; 66 \%$ in $2001 ; 74 \%$ in $2002 ; 82 \%$ in 2003; 74\% in 2004; 76\% in 2005). Appendix Table A1 contains detailed descriptive statistics.

PC-specific medical costs at 24 months followed a rightskewed, nonnormal distribution. No clustering at zero was observed. Censoring rates at 24 months were $22 \%$ for those patients who did not receive any chemotherapy and $17 \%$ for those who received chemotherapy.

\section{Cost-Effectiveness Results}

Mean costs were $\$ 34,973$ (effects, 670 days) and $\$ 21,143$ (effects, 618 days) in the treatment and comparison groups, respectively (Table 1). Incremental costs were \$13,830 (95\% CI, $\$ 11,828$ to $\$ 15,772)$ and incremental effects were 52 days (95\% CI, 40 to 64), resulting in an ICER of $\$ 99,146$ (95\% CI, $\$ 75,042$ to $\$ 130,195)$ per LYG.

Sample sizes across race/ethnicity subgroups included $\mathrm{n}=$ 736, $\mathrm{n}=104$, and $\mathrm{n}=90$ in the treatment groups and $\mathrm{n}=$ $2,249, \mathrm{n}=395$, and $\mathrm{n}=314$ in the comparison groups for non-Hispanic whites, non-Hispanic blacks, and others, respectively. Incremental costs were $\$ 14,355, \$ 10,851$, and $\$ 13,346$ for non-Hispanic whites, non-Hispanic blacks, and others, respectively. Incremental survival effects were 50 days, 71.2 days, and 42.9 days, for non-Hispanic whites, non-Hispanic blacks, and others, respectively, which leads to ICERs (costs per LYG) of $\$ 107,095, \$ 59,887$, and $\$ 123,909$ for non-Hispanic whites, non-Hispanic blacks, and others, respectively. Table 1 reports 95\% CIs for incremental costs, effects, and ICERs.

\section{Sensitivity Analysis}

Sampling uncertainty is evaluated graphically in Figures 1 and 2. Figure 1 depicts a scatter plot of incremental costs and effects according to race/ethnicity subgroups on the basis of 1,000 bootstrap samples per subgroup. Most observations are in the northeast quadrant, which indicates that chemotherapy receipt is associated with increased survival as well as increased PCspecific costs. ICERs for whites are tightly clustered, whereas those for blacks and others exhibit greater variation. The majority of observations for blacks are to the southeast of those for whites, which suggests lower costs and greater effects for blacks.
However, CIs for incremental costs, effects, and ICERs for all three race/ethnicity subgroups overlap (Table 1).

Taking this sampling uncertainty into account, Figure 2 plots the likelihood of chemotherapy being cost-effective for a range of WTP thresholds. For the full sample, this CEAC suggests that, for chemotherapy to be cost-effective in $90 \%$ of patients, society's WTP for an additional life-year would have to be approximately $\$ 120,000$. This masks substantial heterogeneity across subgroups. To be $90 \%$ cost-effective, the required WTP threshold would be $\$ 135,000$ for whites, $\$ 90,000$ for blacks, and $\$ 230,000$ for others. Results from sensitivity analysis of study parameters were similar (Appendix Table A2).

We also examined the effect of selection into treatment on observables by calculating costs and effects in PS-matched subsamples. In the full sample without stratification, the ICER was virtually identical. The ICER for blacks decreased by half, whereas the ICER for others increased by a factor of two. CIs were much wider across all subgroups. In all sensitivity analysis scenarios, CIs overlapped with those from the baseline scenario.

\section{Discussion}

This article reports a series of ICERs for chemotherapy treatment in metastatic PC across demographic subgroups. Our full sample results indicate a cost per LYG of just below the $\$ 100,000$ threshold in the baseline scenario. Point estimates reveal considerable variation across subgroups defined by race/ ethnicity from a low that is not much higher than the $\$ 50,000$ threshold in non-Hispanic blacks to a high that is nearly $\$ 125,000$ for others. Compared with the full sample, CIs for subgroups may be narrower or wider: on the one hand, increased homogeneity within a subgroup would tend to narrow the $\mathrm{CI}$; on the other hand, statistical uncertainty stemming from a smaller sample size may lead to a wider CI. In our sample, the second effect seems to dominate the first, specifically for blacks and others.

Although the estimated ICERs are not statistically different across race/ethnicity groups, uncertainty within subgroups may result in different conclusions regarding cost-effectiveness. At a WTP threshold of $\$ 100,000$ and in a population consisting only of whites, chemotherapy would be cost-effective in only $37.6 \%$ of cases and hence not recommended. Among blacks, however, chemotherapy would be cost-effective in $94 \%$ of cases at that threshold. Uncertainty is greatest for the third race/ ethnicity group, which contains observations of patients with Asian and Hispanic backgrounds in roughly equal proportion-two groups with generally disparate health outcomes. ${ }^{26}$ Although the ICER point estimate in this subgroup is similar to that of whites, the scatter plot shows much more dispersion (Figure 1), and chemotherapy would be cost-effective in $90 \%$ of cases at a much higher WTP threshold of \$230,000 (Figure 2).

Faced with such considerable uncertainty, insurance companies may opt for less favorable coverage or higher cost-sharing, depending on their enrollee population. An alternative approach would be the collection of additional subgroup-specific information to build a more robust evidence base. Efforts to 


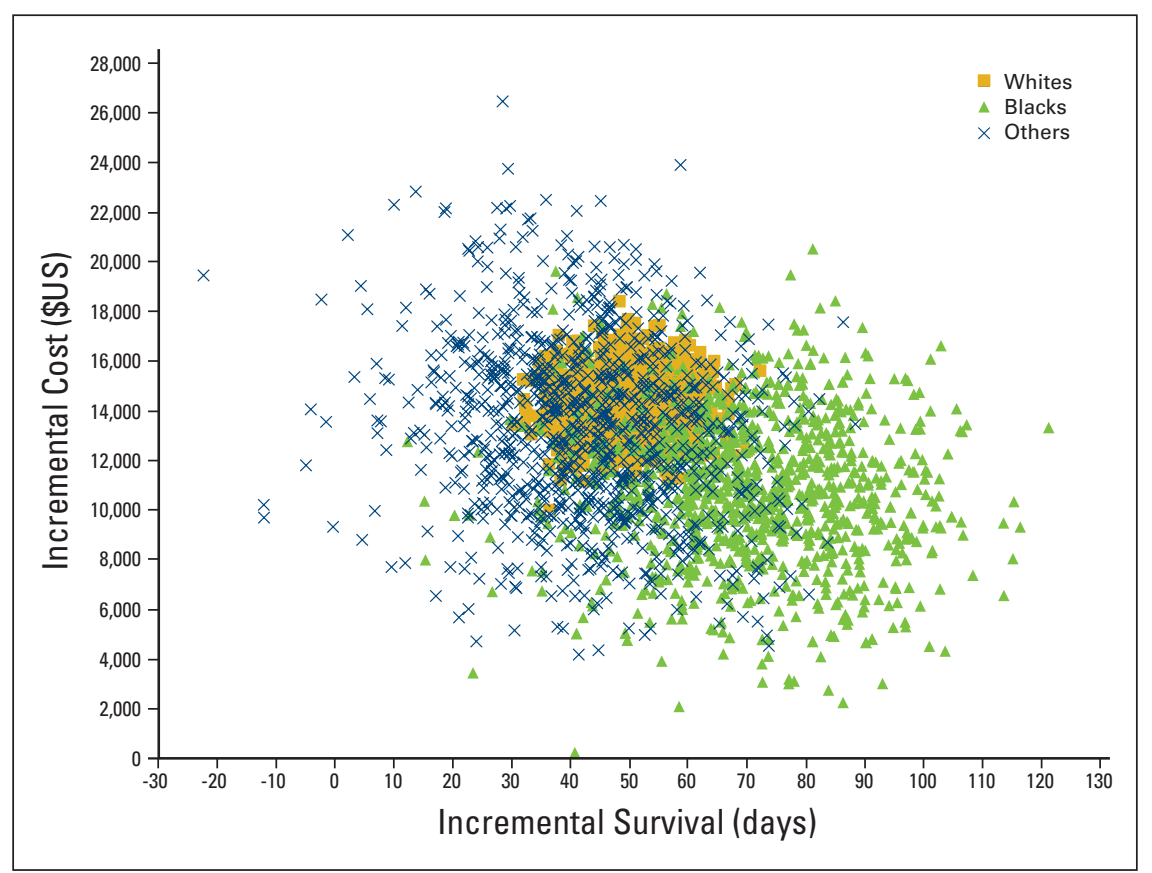

Figure 1. Scatter plot of incremental costs and effects according to race/ethnicity subgroups on the basis of 1,000 bootstrap samples.

increase the representation of minority populations in clinical trials, registries, and other data sets have intensified during the past decade, ${ }^{27,28}$ but barriers remain. ${ }^{29}$ Given the controversies surrounding subgroup analysis, 2,30 there is also a need for guidelines on how to conduct and report the results of such stratification in cost-effectiveness studies, similar to recommendations currently being introduced for clinical trials. ${ }^{31}$

Our study has several advantages. Although the cost-effectiveness of docetaxel-based chemotherapy has been examined previously (with a reported ICER of $£ 32,706[\$ 51,028]$ per quality-adjusted life-year compared with mitoxantrone-based chemotherapy), ${ }^{32}$ that analysis was undertaken using a decision-analytic model and did not examine variation across subgroups. Our study, in contrast, uses patient-level data from a diverse, real-world population and examines cost-effectiveness for important demographic subgroups. Approximately $20 \%$ of observations in our sample were censored; to address the resulting bias in mean cost estimation, we applied inverse probability

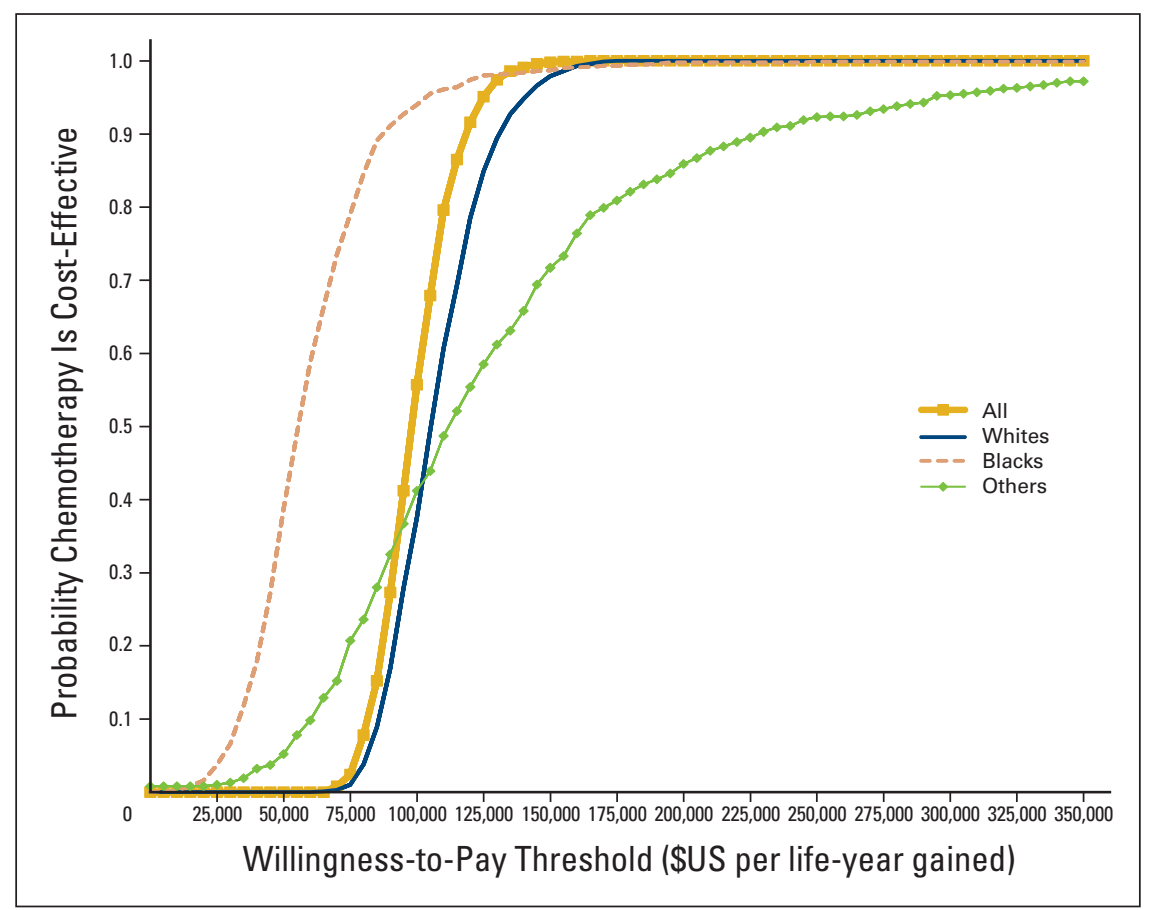

Figure 2. Likelihood of chemotherapy being cost-effective for a range of willingness-to-pay thresholds. 
weighting to the complete observations. Although adjusting for censoring is part of the International Society for Pharmacoeconomics and Outcomes Research cost-effectiveness analysis guidelines, ${ }^{33}$ it is only slowly becoming more widespread in practice. ${ }^{19}$ Finally, we have conducted sensitivity analyses for key alternative scenarios as well as PS matching, to account for selection into treatment, which is a well-known challenge in the use of observational data. ${ }^{34}$

This study also has limitations. We examined elderly patients with PC in SEER-Medicare, and generalizability beyond the Medicare population may be limited. We chose to model the cost-effectiveness of any chemotherapy receipt compared with no chemotherapy receipt as opposed to looking at specific agents because of concerns about selection into agent-specific treatment and reduced statistical power as a result of smaller sample sizes across subgroups. The study is conducted from a payer's point of view and does not include all costs that may be relevant from society's standpoint. In addition, we did not adjust cost and effect estimates for all potential confounders. Covariate adjustment in the presence of censored data is an active research topic. ${ }^{35,36}$ We believe that our focus on patients with M1 disease and stratification by race addressed the key confounding variables in this population; furthermore, results were similar in samples in which patients were matched on the basis of PS.

In conclusion, in a sample of elderly patients with M1 prostate cancer, the ICER of chemotherapy is estimated at $\$ 99,146$ per LYG compared with no chemotherapy. Although point estimates vary across subgroups defined by race/ethnicity, CIs overlap. As a result of substantial statistical uncertainty, especially within minority subgroups, chemotherapy may be considered cost-effective at vastly different WTP thresholds. This suggests that efforts to increase the available information by broadening the representation of minorities in clinical trials, registries, and other health care data sources need to continue.

Accepted for publication on March 25, 2011.

\section{References}

1. Kravitz RL, Duan N, Braslow J: Evidence-based medicine, heterogeneity of treatment effects, and the trouble with averages. Milbank Q 82:661-687, 2004

2. Gabler NB, Duan N, Liao D, et al: Dealing with heterogeneity of treatment effects: Is the literature up to the challenge? Trials 10:43, 2009

3. Sculpher M: Subgroups and heterogeneity in cost-effectiveness analysis. Pharmacoeconomics 26:799-806, 2008

4. Stevens W, Normand C: Optimisation versus certainty: Understanding the issue of heterogeneity in economic evaluation. Soc Sci Med 58:315-320, 2004

5. American Cancer Society: Cancer Facts \& Fiqures 2010. Atlanta. GA. American Cancer Society.2010. http:wwww.cancer.org/acs/aroups/content/@ epidemiologysurveilance/documents/document/acspc-026238.pdf

6. Mullins CD, Onukwugha E, Bikov $\mathrm{K}$, et al: Health disparities in staging of SEER-Medicare prostate cancer patients in the United States. Urology 76:566572, 2010

7. Gilligan $T$, Wang PS, Levin R, et al: Racial differences in screening for prostate cancer in the elderly. Arch Intern Med 164:1858-1864, 2004

8. Zeliadt SB, Potosky AL, Etzioni R, et al: Racial disparity in primary and adjuvant treatment for nonmetastatic prostate cancer: SEER-Medicare trends 1991 to 1999. Urology 64:1171-1176, 2004

9. Do YK, Carpenter WR, Spain P, et al: Race, healthcare access and physician trust among prostate cancer patients. Cancer Causes Control 21:31-40, 2010

\section{Authors' Disclosures of Potential Conflicts of Interest}

Although all authors completed the disclosure declaration, the following author(s) indicated a financial or other interest that is relevant to the subject matter under consideration in this article. Certain relationships marked with a " $U$ " are those for which no compensation was received; those relationships marked with a " $\mathrm{C}$ " were compensated. For a detailed description of the disclosure categories, or for more information about ASCO's conflict of interest policy, please refer to the Author Disclosure Declaration and the Disclosures of Potential Conflicts of Interest section in Information for Contributors.

Employment or Leadership Position: None Consultant or Advisory Role: Eberechukwu Onukwugha, Pfizer (C); C. Daniel Mullins, Bayer Pharmaceuticals (C), Novartis (C), Bristol-Myers Squibb (C), Pfizer (C), Eisai (C), sanofi-aventis (C) Stock Ownership: None Honoraria: None Research Funding: Michael Grabner, sanofi-aventis; Eberechukwu Onukwugha, Novartis, Pfizer, sanofi-aventis; C. Daniel Mullins, Bayer Pharmaceuticals, Novartis, Pfizer, sanofi-aventis Expert Testimony: None Other Remuneration: None

\section{Author Contributions}

Conception and design: Michael Grabner, Eberechukwu Onukwugha, Rahul Jain, C. Daniel Mullins

Financial support: C. Daniel Mullins

Administrative support: Michael Grabner

Provision of study material or patients: C. Daniel Mullins

Collection and assembly of data: Eberechukwu Onukwugha, C. Daniel Mullins

Data analysis and interpretation: Michael Grabner, Eberechukwu Onukwugha, Rahul Jain, C. Daniel Mullins

Manuscript writing: Michael Grabner, Eberechukwu Onukwugha, Rahul Jain, C. Daniel Mullins

Final approval of manuscript: Michael Grabner, Eberechukwu Onukwugha, Rahul Jain, C. Daniel Mullins

Corresponding author: Michael Grabner, PhD, Department of Pharmaceutical Health Services Research, University of Maryland School of Pharmacy, 220 Arch Street, 12th Floor, Baltimore, MD 21201; e-mail: mjgrabner@gmail.com.

DOI: 10.1200/JOP.2011.000294

10. Powell IJ: Epidemiology and pathophysiology of prostate cancer in AfricanAmerican men. J Urol 177:444-449, 2007

11. Sternberg CN: Highlights of contemporary issues in the medical management of prostate cancer. Crit Rev Oncol Hematol 43:105-121, 2002

12. Dagher R, Li N, Abraham S, et al: Approval summary: Docetaxel in combination with prednisone for the treatment of androgen-independent hormone-refractory prostate cancer. Clin Cancer Res 10:8147-8151, 2004

13. Lassi K, Dawson NA: Update on castrate-resistant prostate cancer: 2010. Curr Opin Oncol 22:263-267, 2010

14. Michael A, Syrigos K, Pandha H: Prostate cancer chemotherapy in the era of targeted therapy. Prostate Cancer Prostatic Dis 12:13-16, 2009

15. Bach PB: Limits on Medicare's ability to control rising spending on cancer drugs. N Engl J Med 360:626-633, 2009

16. Greene FL, Page DL, Fleming ID, et al: AJCC Cancer Staging Manual (ed 6). New York, NY, Springer, 2002

17. Brown ML, Riley GF, Schussler N, et al: Estimating health care costs related to cancer treatment from SEER-Medicare data. Med Care 40:IV-104-117, 2002 (suppl)

18. Desgagné A, Castilloux AM, Angers JF, LeLorier J: The use of the bootstrap statistical method for the pharmacoeconomic cost analysis of skewed data. Pharmacoeconomics 13:487-497, 1998 
19. Doshi JA, Glick HA, Polsky D: Analyses of cost data in economic evaluations conducted alongside randomized controlled trials. Value Health 9:334-340, 2006 20. Bang H, Tsiatis AA: Estimating medical costs with censored data. Biometrika 87:329-343, 2000

21. Zhao $\mathrm{H}$, Wang $\mathrm{H}$ : Cost and cost-effectiveness analysis with censored data, in Faries D, Leon A, Haro J, et al (ed): Analysis of Observational Health Care Data Using SAS. Cary, NC, SAS Institute, 2010

22. Chaudhary MA, Stearns SC: Estimating confidence intervals for cost-effectiveness ratios: An example from a randomized trial. Stat Med 15:1447-1458, 1996

23. Briggs AH, O'Brien BJ, Blackhouse G: Thinking outside the box: Recent advances in the analysis and presentation of uncertainty in cost-effectiveness studies. Annu Rev Public Health 23:377-401, 2002

24. Rosenbaum P, Rubin D: The central role of the propensity score in observational studies for causal effects. Biometrika 70:41-55, 1983

25. Parsons LS: Reducing bias in a propensity score matched-pair sample using greedy matching techniques. Presented at the 26th Annual SAS Users' Group International Conference. Long Beach, CA, April 22-25, 2001 (abstr 214-26)

26. Keppel K, Garcia T, Hallquist S, et al: Comparing racial and ethnic populations based on Healthy People 2010 objectives. Healthy People Stat Notes 2008:1-16, 2008

27. Mullins $\mathrm{CD}$, Onukwugha E, Cooke JL, et al: The potential impact of comparative effectiveness research on the health of minority populations. Health Aff (Millwood) 29:2098-2104, 2010

28. Bruner DW, Jones M, Buchanan D, et al: Reducing cancer disparities for minorities: A multidisciplinary research agenda to improve patient access to health

\section{Appendix}

\section{Exclusion Criteria}

Patients were excluded if one or more of the following applied: (1) age less than 66 years, (2) history of cancer in the previous 5 years, (3) cancer diagnosis from death certificate or autopsy, (4) unknown diagnosis month, (5) not enrolled in Medicare Part A and $B$ for the 12 months before and including the month of diagnosis, (6) enrolled in a health maintenance organization for the 12 months before and including the month of diagnosis, (7) unknown Surveillance, Epidemiology, and End Results (SEER) stage.

\section{Propensity Score Model}

The following variables were included in the propensity score (PS) model for the full sample: age group at diagnosis (referent category $[\mathrm{RC}]$, age 66-74 years), race/ethnicity (RC, non-His- systems, clinical trials, and effective cancer therapy. J Clin Oncol 24:2209-2215, 2006

29. Ford JG, Howerton MW, Lai GY, et al: Barriers to recruiting underrepresented populations to cancer clinical trials: A systematic review. Cancer 112:228-242, 2008

30. Cook DI, Gebski VJ, Keech AC: Subgroup analysis in clinical trials. Med $J$ Aust 180:289-291, 2004

31. Kent DM, Rothwell PM, loannidis JP, et al: Assessing and reporting heterogeneity in treatment effects in clinical trials: A proposal. Trials 11:85, 2010

32. Collins R, Fenwick E, Trowman R, et al: A systematic review and economic model of the clinical effectiveness and cost-effectiveness of docetaxel in combination with prednisone or prednisolone for the treatment of hormone-refractory metastatic prostate cancer. Health Technol Assess 11:iii-iv, xv-xviii, 1-179, 2007

33. Ramsey S, Willke R, Briggs A, et al: Good research practices for cost-effectiveness analysis alongside clinical trials: The ISPOR RCT-CEA Task Force report. Value Health 8:521-533, 2005

34. Johnson ML, Crown W, Martin BC, et al: Good research practices for comparative effectiveness research: Analytic methods to improve causal inference from nonrandomized studies of treatment effects using secondary data sourcesThe ISPOR Good Research Practices for Retrospective Database Analysis Task Force report, part III. Value Health 12:1062-1073, 2009

35. Willan AR, Lin DY, Manca A: Regression methods for cost-effectiveness analysis with censored data. Stat Med 24:131-145, 2005

36. Basu A, Manning WG: Estimating lifetime or episode-of-illness costs under censoring. Health Econ 19:1010-1028, 2010

panic white), currently married (RC, not currently married), poorly differentiated tumor (RC, well or moderately differentiated tumor), Charlson comorbidity index (CCI) score (RC, CCI score is 0), positive prostate-specific antigen (PSA) test (RC, nonpositive PSA test), patient residence in an urban location ( $\mathrm{RC}$, nonurban residence), and a proxy measure of poor performance status based on whether or not the patient had a claim during the 12 months prediagnosis indicating the use of a wheelchair, a walking aid, home oxygen, a skilled nursing facility, or a hospitalization. The PS model also contained census-level measures of socioeconomic status (\% of persons with 4 or more years of college education; \% of households speaking English not well or not at all; median income for the census tract) and indicators for diagnosis years and the locations of SEER registries. 
Table A1. Characteristics of Patients With Metastatic Prostate Cancer on ADT With a Minimum Survival of 3 Months (N = 3,888)

\begin{tabular}{|c|c|c|c|c|c|c|c|c|c|c|}
\hline Characteristic & \multicolumn{5}{|c|}{ Any Chemotherapy } & \multicolumn{5}{|c|}{ No Chemotherapy } \\
\hline \multicolumn{11}{|l|}{ Demographic } \\
\hline Mean age, years & 74.6 & 74.8 & 74.1 & 73.4 & .13 & 78.6 & 79.2 & 76.1 & 77.5 & $<.001$ \\
\hline \multicolumn{11}{|l|}{ Clinical } \\
\hline $\begin{array}{l}\text { Poorly differentiated } \\
\text { tumor, \% }\end{array}$ & 63.6 & 62.4 & 69.2 & 66.7 & .32 & 56.5 & 55.9 & 57.0 & 60.5 & .29 \\
\hline $\mathrm{CCl} \geq 2, \%$ & 10.0 & 9.7 & 11.5 & 11.1 & .78 & 15.1 & 14.5 & 17.7 & 15.9 & .23 \\
\hline \multicolumn{11}{|l|}{ Treatment } \\
\hline Docetaxel, \% & 72.4 & 72.0 & 74.0 & 73.3 & .89 & & & & & \\
\hline Radiation, \% & 60.2 & 62.2 & 46.2 & 60.0 & .01 & 33.9 & 34.4 & 26.1 & 39.8 & .00 \\
\hline Urologist visit, \% & 87.2 & 87.8 & 87.5 & 82.2 & .33 & 86.6 & 86.5 & 88.1 & 85.7 & .60 \\
\hline RadOnc visit, \% & 40.7 & 42.1 & 29.8 & 41.1 & .06 & 26.5 & 26.5 & 22.5 & 32.2 & .02 \\
\hline MedOnc visit, \% & 85.5 & 86.7 & 77.9 & 84.4 & .06 & 49.5 & 49.8 & 50.1 & 46.5 & .53 \\
\hline Primary care visit, \% & 62.5 & 64.5 & 50.0 & 60.0 & .01 & 65.7 & 66.7 & 62.8 & 61.8 & .10 \\
\hline \multicolumn{11}{|l|}{ Other } \\
\hline Urban domicile, \% & 91.0 & 90.0 & 91.4 & 98.9 & .02 & 87.0 & 84.7 & 91.7 & 97.8 & $<.001$ \\
\hline Poor performance, \%* & 18.0 & 18.5 & 17.3 & 14.4 & .63 & 24.1 & 24.0 & 27.3 & 21.3 & .17 \\
\hline PSA test, \% & 83.2 & 83.6 & 78.9 & 85.6 & .40 & 82.3 & 82.4 & 82.3 & 81.5 & .92 \\
\hline Positive PSA test, \% & 79.7 & 79.4 & 77.9 & 84.4 & .47 & 79.9 & 80.0 & 80.3 & 79.3 & .95 \\
\hline
\end{tabular}

NOTE. $P$ values are from $\chi^{2}$-tests for categorical variables and rank sum tests for continuous variables.

Abbreviations: ADT, androgen deprivation therapy; CCI, Charlson Comorbidity Index; MedOnc, medical oncologist; PSA, prostate-specific antigen; RadOnc, radiation oncologist.

* At least one indicator of poor performance status (presence of a claim for use of a wheelchair, a walking aid, home oxygen, a skilled nursing facility, or a hospitalization during the 12 months prior to diagnosis). 
Table A2. Sensitivity Analysis

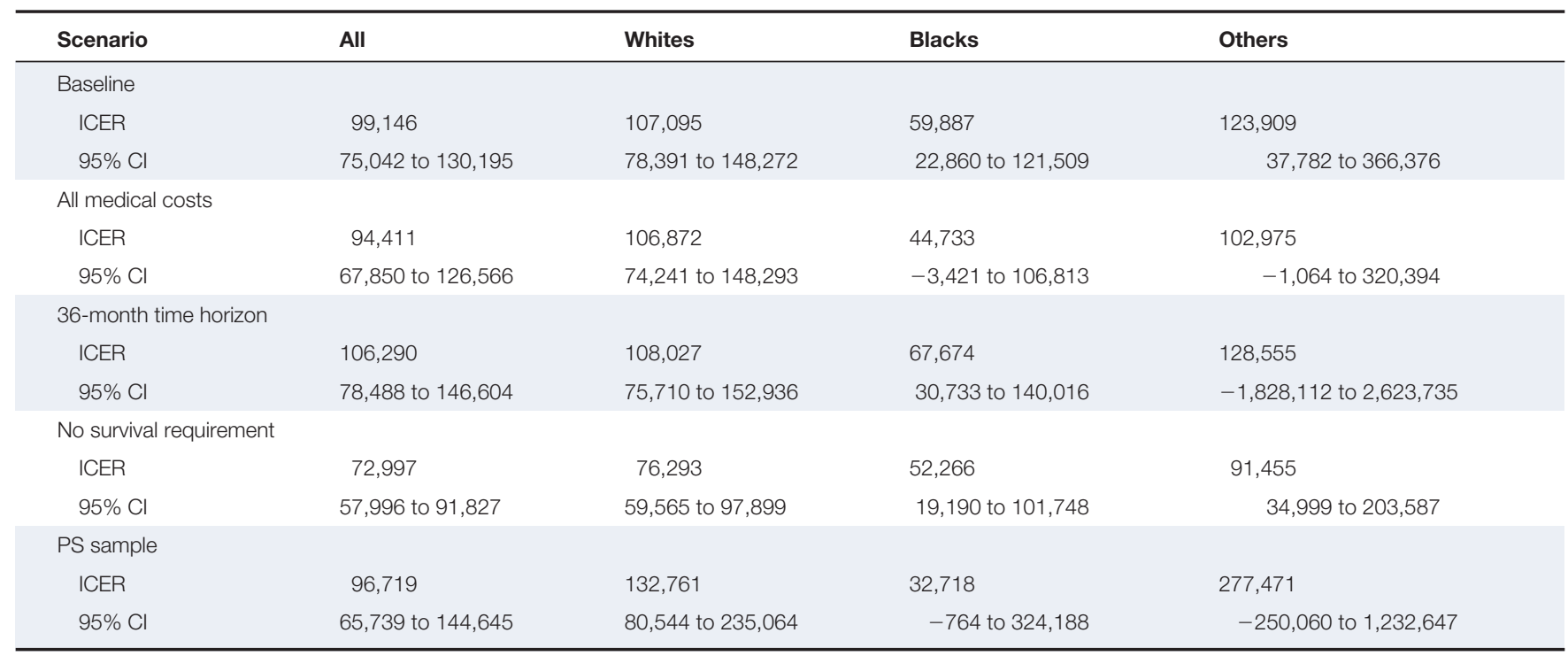

NOTE. ICERs are reported in \$US per LYG and are based on a bootstrap with 1,000 replications. Cls were calculated using a percentile method from the same bootstrap. Baseline scenario reflects 3 months minimum survival from diagnosis, prostate cancer-related costs, and a 24-month time horizon. Some Cls contain negative ICERs as a result of a few bootstrap replications with negative incremental costs or negative incremental effects. There were no bootstrap replications with both negative incremental costs and negative incremental effects.

Abbreviations: ICER, incremental cost-effectiveness ratio; LYG, life-year gained; PS, propensity score. 